Background There are increasing numbers of children with palliative care needs in the community. The death of a child is a rare but tragic event. Currently a considerable number of children who do die, do so on PICU. End-of-life care and ACP in paediatric patients presents one of the most complex, emotional and ethically challenging scenarios that exists in clinical medicine. Currently there is a lack of uniformity in approach.

Aim The overall aim of the project was to investigate the research question: "What is current practise related to ACP in relation to children with life-limiting illness on PICU, and how might this be improved?"

Methods Qualitative semi-structured interviews were conducted with senior medical and nursing staff in a tertiary referral centre PICU. Interviews were transcribed, field notes were documented and a thematic content analysis was carried out, using an inductive approach.

Results The sample comprised eight consultants and six senior nurses. Themes were revealed around "the significance of the death of a child". ACP is a dynamic, MDT process that is considered essential in order to improve care for children with life-limiting conditions to increase the likelihood of "a good death". Every case is different, but PICU clinicians feel that ACP can be associated with the delivery of appropriate care that is in the best interests of the patient. Multiple barriers exist, including the difficulty in raising the issue, unpredictable illness trajectories making it difficult to identify when to commence discussions, multicultural issues, and lack of training in and time to deliver ACP.

Conclusion "How people die remains in the memory of those who live on." Dame Cicely Saunders.

The motivations for improvement of ACP for paediatric patients with palliative care needs are numerous, nationally to standardise care and ensure appropriate use of pressurised NHS resources, locally within regions to provide care appropriate for the population and close to home, and most of all on an individual level to ensure the best possible care, with respect for autonomy and choice, for each child with a life-limiting illness and their family.

\section{G42(P) NATIONAL SURVEY OF THE USE OF FORMAL ADVANCE CARE PLAN (ACP) DOCUMENTS FOR PAEDIATRIC PATIENTS WITH LIFE-LIMITING CONDITIONS IN UK PAEDIATRIC INTENSIVE CARE UNITS (PICUS)}

doi:10.1136/archdischild-2013-304107.054

1,2,3,4S Mitchell, 'A Plunkett, '2J Dale. 'PICU, Birmingham Children's Hospital, Birmingham, UK; ${ }^{2}$ Health Sciences, University of Warwick, Coventry, UK; ${ }^{3}$ Oncology and Palliative Medicine, University of Cardiff, Cardiff, UK; ${ }^{4}$ Clinical Innovation and Research Centre, Royal College of General Practitioners, London, UK

Background There are few clinical scenarios as difficult, emotive and ethically challenging as those surrounding the end-of-life care of children with life-limiting illness. Intensive care staff, who are at the forefront of developing and utilising life-sustaining technologies and interventions, inevitably become responsible for the management of the end-of-life once intensive treatment options have been exhausted or prove to be futile.

ACP has been defined as a process of discussion between an individual, their care providers, and often those close to them, about future care. Formal ACP documents are currently being advocated in order to provide structure and to improve the ACP process. Currently uptake varies both within regional and national centres.

Aim To conduct a survey of current ACP documents in use within secondary care settings (hospitals with PICU) in the UK.

Method An email survey was sent to lead clinicians from the 28 PICUs in the UK whose contact details were obtained using the "Paediatric Intensive Care Audit Network (PICANet) list of unit contacts 2011". Non-responders were emailed again one, two and three months later.
Results 24 replies (85.71\%) were received. 14 (58.33\%) of the 24 PICUs who replied to the survey have ACP documents in use. Three departments use the "Wishes" documents. Other ACPs in use were the WMPPCN Paediatric Palliative Care Toolkit ACP and the South Central ACP.

Of the ten units who do not currently have formal ACP documents, one detailed plans to launch a local document in the near future, and three listed either rapid discharge and extubation pathways or deterioration management pathways that are currently in use.

Conclusion The motivations for ACP for children with life-limiting illness are numerous, and include recognition that intensive therapy and invasive interventions may be inappropriate when death is near or inevitable. ACP potentially allows the facilitation of choice for patients and their families. Guidelines, strategies and policies for ACP are being produced faster than the evidence base to support them. This may currently be a barrier to uptake of formal ACP documents, and warrants further investigation.

\section{G43(P) CLINICAL AUDIT ON ADVANCED CARE PLAN FOR MANAGEMENT OF CARDIO-RESPIRATORY ARREST IN CHILDREN AND YOUNG PERSON WITH ADVANCED MALIGNANCY (SINGLE CENTRE EXPERIENCE)}

doi:10.1136/archdischild-2013-304107.055

${ }^{1} \mathrm{M}$ Aye, ${ }^{2} \mathrm{D}$ Hobin. ${ }^{1}$ Paediatric Department, UHNS, Stoke on Trent, UK; ${ }^{2}$ Paediatric Oncology Department, BCH, Birmingham, UK

Aim To find out whether children with advanced malignant disease referred to Palliative care team had advanced care plan for management of cardiopulmonary arrest or not.

Secondary objectives To detect designated purple form (Advance care plan-ACP) from the West midland Paediatric palliative care toolkits have been used or not.

To identify the heath care profession who involved in making ACP decision.

Lastly, to assess place of death related to presence of ACP with DNAR (Do Not Attempt Resuscitation).

Methods Retrospective study of Children with advanced malignant disease who died while under the care of Palliative care team (BCH) during May to October 2011.

Data were collected from Palliative care data base, Patients' notes (medical and palliative care notes) and Letters.

Standard Child \& Young Person's Advance Care Plan Policy - April 2011West Midlands Paediatric Palliative Care Network, Paediatric Palliative Care toolkit

Results Total number of death from June 2011 to Oct 2011 was 26, 23 with solid Tumour and 3 with haematology malignancy. Age ranged from neonate till 16 years.

Eighty percent (21/26) of children had formulated plan for DNAR. Half (51\%) used the purple form (ACP from West midland Paediatric Palliative care toolkit), the remaining half either by using other DNAR forms $(20 \%)$ or letters $(28 \%)$.

Most $(90 \%)$ of the ACP with DNAR were supported by consultant (paediatric oncologist) and only 10\% was by general practitioner.

Eighteen children (68\%) died at home and all of them had ACP with DNAR. 2 children with ACP with DNAR, died at hospice. $26 \%$ (6) died in hospital (oncology ward/PICU), 5 children without ACP and 1 child with ACP in place. All the children (3) with haematological malignancy died in hospital while they were on active treatment without ACP in place.

Conclusion Advanced care plan discussion should start early in the course of illness according to the pace of parents and children at the sensitive manner. The regional standard ACP form (Purple form-Palliative care toolkits) should be used in all cases with life limiting conditions. 